\title{
The Study on the Convex and LMI Conditions for Static Output Feedback Stabilization
}

\author{
Pang-Chia Chen \\ Assistant Professor, Department of Aircraft Engineering, Air Force Institute of Technology, Kangshan County, Taiwan \\ 820, R.O.C.
}

\section{Koan-Yuh Chang}

Assistant Professor, Department of Electronic Engineering, Chien Kuo Institute of Technology, Changhua City, Taiwan 500, R.O.C.

\section{Yaug-Fea Jeng}

Assistant Professor, Department of Aircraft Engineering, Air Force Institute of Technology, , Kangshan County, Taiwan 820, R.O.C.

\author{
Wen-Jer Chang \\ Professor, Department of Marine Engineering, National Taiwan Ocean University, Keelung, Taiwan 202, R.O.C., \\ wjchang@mail.ntou.edu.tw
}

Follow this and additional works at: https://jmstt.ntou.edu.tw/journal

Part of the Engineering Commons

\section{Recommended Citation}

Chen, Pang-Chia; Chang, Koan-Yuh; Jeng, Yaug-Fea; and Chang, Wen-Jer (2004) "The Study on the Convex and LMI Conditions for Static Output Feedback Stabilization," Journal of Marine Science and Technology. Vol. 12: Iss. 3, Article 5 . DOI: $10.51400 / 2709-6998.2235$

Available at: https://jmstt.ntou.edu.tw/journal/vol12/iss3/5

This Research Article is brought to you for free and open access by Journal of Marine Science and Technology. It has been accepted for inclusion in Journal of Marine Science and Technology by an authorized editor of Journal of Marine Science and Technology. 


\title{
THE STUDY ON THE CONVEX AND LMI CONDITIONS FOR STATIC OUTPUT FEEDBACK STABILIZATION
}

\author{
Pang-Chia Chen*, Koan-Yuh Chang**, Yaug-Fea Jeng***, and Wen-Jer Chang****
}

Key words: static output-feedback stabilization, convex condition, LMI.

\section{ABSTRACT}

In this paper, the problem of static output feedback stabilization is considered. By congruence and state-coordinate transformations, a convex condition for static output feedback stabilization is given in terms of a matrix linear inequality while subject to an equality constraint. To facilitate stabilization design via LMI conditions, the equality constraint is satisfied by imposing a structure constraint on matrix variable. Moreover, a numerical construction algorithm and a sufficient condition for the presented convex condition are given as well. Finally, numerical examples demonstrated the utility of the proposed approach for static output feedback stabilization.

\section{INTRODUCTION}

The static output feedback problem concerns finding a static or constant feedback gain to achieve certain desired closed-loop characteristics. The output feedback control problem is much more difficult to solve when compared to state feedback control problem. Though many important control problems using state feedback can be solved via linear matrix inequality (LMI) techniques [3], this is not the case for the available design techniques for output feedback control; if on one hand the existing convex approaches are based on sufficient conditions they may be restrictive, on the other hand the approaches based on necessary and sufficient condition are not numerical tractable $[12,15]$.

A variety of static output feedback problems were studied by many researchers with analytical and numerical methods, for example, the comprehensive sur-

Paper Submitted 02/27/04, Accepted 06/02/04. Author for Correspondence: Wen-Jer Chang.E-mail: wjchang@mail.ntou.edu.tw.

*Assistant Professor, Department of Aircraft Engineering, Air Force Institute of Technology, Kangshan County, Taiwan 820, R.O.C.

**Assistant Professor, Department of Electronic Engineering, Chien Kuo Institute of Technology, Changhua City, Taiwan 500, R.O.C.

***Assistant Professor, Department of Aircraft Engineering, Air Force Institute of Technology, , Kangshan County, Taiwan 820, R.O.C.

****Professor, Department of Marine Engineering, National Taiwan Ocean University, Keelung, Taiwan 202, R.O.C. vey given in $[2,14]$ One approach is based on the fact that the existence of a static output feedback gain is equivalent to a positive definite matrix variable $Q$ satisfying simultaneously two Lyapunov inequalities, where one inequality related with $Q$ and the other with $Q^{-1}$ [11]. In order to handle this matrix inversion condition, various iterative schemes have been developed such as the rank minimization algorithm [6], the min-max algorithm [8], and the product reduction algorithmn [9]. Another iterative approach presented in [4] was derived based on a necessary and sufficient condition. As for the non-iterative approach, a sufficient condition for static output control was given in [5] for systems with measurement matrix $C$ full row rank or input matrix $B$ full column rank. In [13], another non-iterative approach is presented for a certain class of plants with matrix product $C B$ full row rank.

In this paper, a convex condition of static output feedback stabilization is proposed for system with the measurement matrix $C$ full row rank or the input matrix $B$ full column rank. The convex conditon are expressed as an LMI condition subject to an equality constraint. The equality constraint is established sufficiently by a Lyapunov matrx $Q$ with block digonal structure. Then, a numerical construction algorithm and a sufficient condition via LMI method for the proposed convex condition for static output feedback stabilization are given. Finally, two numerical examples are presented to demonstrate the capability of the proposed approaches.

\section{CONVEX AND LMI CONDITIONS}

Consider a linear time invariant system $G$ described by the state-space equations

$$
G:\left\{\begin{array}{l}
\dot{x}=A x+B u, \\
u=C x
\end{array}\right.
$$

where $x \in R^{n}$ is the state, $u \in R^{m}$ is the control input, and $y \in R^{p}$ is the measurement. It is assumed that the matrix pairs $(A, B)$ and $(A, C)$ are assumed to be, respectively, stabilizable and detectable, and the rank conditions 
either $\operatorname{rank}(C)=p$ or $\operatorname{rank}(B)=m$. The aim is to compute a static output feedback law $u=F y$ such that the stability of the closed-loop syetm $A_{c l}=A+B F C$ is ensured. Therefore, the closed-loop system must admit a symmetric Lyapunov function $P>0$ such that

$$
\begin{aligned}
Z: & =A_{c l}^{T} P+P A_{c l} \\
& =A^{T} P+P A+C^{T} F^{T} B^{T} P+P B F C .
\end{aligned}
$$

By denoting the square and non-singular matrix $\hat{C}^{T}=\left[C^{T} T_{c}^{T}\right]$ with $T_{c}$ chosen to be an orthogonal basis of the null space of the full row rank output matrix $C$, the equation (2) can be written as

$$
Z=A^{T} P+P A+\hat{C}^{T}\left[\begin{array}{ll}
F & 0
\end{array}\right]^{T} B^{T} P+P B\left[\begin{array}{ll}
F & 0
\end{array}\right] \hat{C}<0,
$$

By using the denotation $P^{-1}:=Q$ and the congruence transformation $Q Z Q$, we have the equivalent stabilizing conditon

$$
\begin{aligned}
Z_{Q}: & =Q Z Q=Q A^{T}+A Q+Q \hat{C}^{T}\left[\begin{array}{ll}
F & 0
\end{array}\right]^{T} B^{T} \\
& +B\left[\begin{array}{ll}
F & 0
\end{array}\right] \hat{C} Q<0 .
\end{aligned}
$$

The following proposition gives a convex constrained condition for the numerical construction of (4). Proposition 1 System (1) with matrix $C$ full row rank is stabilizable by static output feedback $u=F y$ if there exist matrices $Q>0, L \in R^{m \times p}$ such that

$$
\begin{aligned}
& Z_{d}=Q_{d} \hat{A}^{T}+\hat{A} Q_{d}+\left[\begin{array}{ll}
L & 0
\end{array}\right]^{T} \hat{B}^{T}+\hat{B}\left[\begin{array}{ll}
L & 0
\end{array}\right]<0, \\
& C Q T_{c}^{T}=0,
\end{aligned}
$$

where $Q_{d}=\hat{C} Q \hat{C}^{T}, \hat{A}=\hat{C} A \hat{C}^{-1}, \hat{B}=\hat{C} B$. Moreover, when the conditions (5) and (6) are feasible, a stabilizing static output feedback is $F=L\left(C Q C^{T}\right)^{-1}$.

Proof Premultiplying and postmultiplying the matrix inequality (4) by $\hat{C}$ and $\hat{C}^{T}$, and according to the definition of $Q_{d}, \hat{A}$, and $\hat{B}$, we have

$$
\begin{aligned}
Z_{\hat{C} Q}: & =\hat{C} Q Z Q \hat{C}^{T} \\
& =\hat{C} Q A^{T} \hat{C}^{T}+\hat{C} A Q \hat{C}^{T}+\hat{C} Q \hat{C}^{T}\left[\begin{array}{ll}
F & 0
\end{array}\right]^{T} B^{T} \hat{C}^{T} \\
& +\hat{C} B\left[\begin{array}{ll}
F & 0
\end{array}\right] \hat{C} Q \hat{C}^{T} \\
& =Q_{d} \hat{A}^{T}+\hat{A} Q_{d}+Q_{d}\left[\begin{array}{ll}
F & 0
\end{array}\right]^{T} \hat{B}^{T}+\hat{B}\left[\begin{array}{lll}
F & 0
\end{array}\right] Q_{d}<0 .
\end{aligned}
$$

If matrix $Q$ satisfies the equality constraint
$C Q T_{c}^{T}=0$ in (6), the matrix $Q_{d}$ is shown to be diagonal, $Q_{d}=\hat{C} Q \hat{C}^{T}=\operatorname{diag}\left(C Q C^{T}, T_{c} Q T_{c}^{T}\right)$. Then, by the denotation $[F 0] Q_{d}=\left[F\left(C Q C^{T}\right) 0\right]:=[L 0]$, we have the matrix inequality (5) which is linear in the matrix variables $L$ and $Q$. The resulting static output feedback gain is $F=L\left(C Q C^{T}\right)^{-1}$. This completes the proof.

The constraint of matrix equation $C Q T_{c}^{T}=0$ in (6) is not readily to be solved by using the MATLAB LMI Toolbox [7]. A sufficient condition for (6) is to find a matrix $M \in R^{p \times p}$ such that

$$
C Q=M C
$$

which was also presented in [5] for derivation of the static output feedback stabilization in a different way of formulation. Then we have $C Q T_{c}^{T}=M C T_{c}^{T}=0$, automatically. In [10], the matrix equality (8) is shown to be equivalent to imposing a block diagonal structural constraint on the matrix variable $Q$. For a full row rank output matrix $C$, the singular value decomposition of $C$ can be represented as

$$
C=U\left(C_{0} 0\right) V^{T}
$$

with $U \in R^{p \times p}$ and $V \in R^{n \times n}$ unitary matrices and $C_{0} \in$ $R^{p \times p}$ a diagonal matrix with positive diagonal elements in decreasing order. This equivalent condition on matrix equation $C Q=M C$ is restated as the following lemma.

Lemma 1 For the given output matrix $C \in R^{p \times n}$ with $\operatorname{rank}(C)=p$ and the symmetric matrix $Q \in R^{n \times n}$, there exists a matrix $M \in R^{p \times p}$ such that $C Q=M C$ if and only if $Q$ can be represented as

$$
Q=V\left(\begin{array}{cc}
Q_{1} & 0 \\
0 & Q_{2}
\end{array}\right) V^{T}
$$

where $Q_{1} \in R^{p \times p}$ and $Q_{2} \in R^{(n-p) \times(n-p)}$. Moreover, the matrix $\mathrm{M}$ is found to be $M=U C_{0} Q_{1} C_{0}^{-1} U^{T}$.

The structural constraint (10) as well as the equality condition (8) on $Q$ is a sufficient condition and shows conservativeness. Indeed, for the case of a partial state measurement, $C=\left[\begin{array}{ll}I_{p} & 0\end{array}\right]$ and $T_{c}^{T}=\left[\begin{array}{ll}0 & I_{n-p}\end{array}\right]^{T}$, the matrix equation (6), $C Q T_{c}^{T}=0$ is exactly equivalent to the block diagonal structural constraint matrix on $Q$, that is, $Q=\operatorname{diag}\left(Q_{1}, Q_{2}\right)$, which is the same as the statement in Lemma 1. However, in order to relieve this diagonal structure constraint for the considered system (1) in a general form, a heuristic construction for the stabilizing static output control law $u=F y$ is given in the following algorithm.

Algorithm 1 Given the system (1) with output matrix $C$ full row rank to be stabilized by the static output feedback, $u=F y$. 
Step 1: Pick the small positive number $\varepsilon_{i, j}, i=1, \ldots, p$, and $j=1, \ldots, n-p$, for the magnitude bound of the elements of matrix product $C Q T_{c}^{T}(i, j)$.

Step 2: Solve for $Q>0$ and L by minimize $\alpha$ subject to the following LMI constraints

$$
\begin{aligned}
& Z_{d}-2 \alpha Q_{d}<0, \\
& -\varepsilon_{i, j}<r_{i} C Q T_{c}^{T} l_{j}^{T}<\varepsilon_{i, j},
\end{aligned}
$$

where $r_{i} \in R^{1 \times p}$ with the $\mathrm{i}$-th element equals to one and others equal to zeros, $l_{j} \in R^{1 \times(n-p)}$ with the $j$-th element equals to one and others equal to zeros. Denote $\alpha^{*}$ as the achieved minimum value of $\alpha$.

Step 3: If $\alpha^{*} \geq 0$, go to Step 6 .

Step 4: If $\alpha^{*}<0$, compute the static output feedback gain $F=L\left(C Q C^{T}\right)^{-1}$. If $Z_{\hat{C} Q}$ in (7) is negative definite, the resulting $F$ is a stabilizing static output feedback gain. Stop.

Step 5: If $\varepsilon_{i, j}<\delta_{\varepsilon}$ for the prescribed tolerance $\delta_{\varepsilon}$, go to Step 6, else choose smaller number for $\varepsilon_{i, j}$, then go to Step 2.

Step 6: the system (1) may not be static output feedback stabilizable. Stop.

The Algorithm 1 is intuitively simple in the process but needs iteration. Alternatively, the following proposition gives a sufficient condition in terms of LMI constraints by relieving the equality condition $C Q T_{c}^{T}=0$ in Proposition 1 as $\left\|C Q T_{c}^{T}\right\|<\varepsilon$ for a small positive $\varepsilon$.

Proposition 2 System (1) is stabilizable by static output feedback with $F=L\left(C Q C^{T}\right)^{-1}$ if for a given small positive number $\varepsilon$, there exist matrices $Q>0, L \in R^{m \times p}$ and free parameter $\xi$ such that the following LMI conditions hold

$$
\begin{aligned}
& C Q C^{T}>I_{p},\left(\begin{array}{cc}
-e I_{n-p} & T_{c} Q C^{T} \\
C Q T_{c}^{T} & -e I_{p}
\end{array}\right)<0, \\
& \left(\begin{array}{cc}
-\xi I_{p} & L^{T} \\
L & -I_{m}
\end{array}\right)<0, \\
& Z_{d}+2 e \xi\|\hat{B}\| I_{n}<0 .
\end{aligned}
$$

Proof From matrix inequality (7) with $F=\mathrm{L}\left(C Q C^{T}\right)^{-1}$, we have

$$
\begin{aligned}
Z_{\hat{C} Q} & =Z_{d}+\left[\begin{array}{ll}
0 & \hat{B} L\left(C Q C^{T}\right)^{-1} C Q T_{c}^{T}
\end{array}\right] \\
& +\left[\begin{array}{ll}
0 & \left(\hat{B} L\left(C Q C^{T}\right)^{-1} C Q T_{c}^{T}\right)^{T}
\end{array}\right] .
\end{aligned}
$$

In case that the equality condition $C Q T_{c}^{T}=0$ holds as described in (6), the equation (15) simply leads to
$Z_{\hat{C} Q}=Z_{d}$. In the general case, we have the following inequality

$$
Z_{\hat{C} Q}<Z_{d}+2\|\hat{B}\|\|L\|\left\|\left(C Q C^{T}\right)^{-1}\right\|\left\|C Q C_{c}^{T}\right\| I_{n} .
$$

By the LMI condition (13), we have

$$
\underline{\lambda}\left(C Q C^{T}\right)>1,\left\|C Q T_{c}^{T}\right\|<\varepsilon,\|L\|<\xi,
$$

where $\underline{\lambda}(\cdot)$ denotes for smallest eigenvalue. Let the symmetric matrix $C Q C^{T}$ represented as $C Q C^{T}=\prod \Lambda \prod^{T}$, where $\Lambda$ is a diagonal matrix containing the eigenvalues of $C Q C^{T}$ and $\prod$ is a unitary matrix with columns made up of the eigenvectors of $C Q C^{T}$. We have,

$$
\begin{aligned}
& \left\|\left(C Q C^{T}\right)^{-1}\right\|=\bar{\sigma}\left(\Pi \Lambda^{-1} \Pi^{T}\right)=\bar{\sigma}\left(\Lambda^{-1}\right)=\frac{1}{\underline{\sigma}(\Lambda)} \\
& =\frac{1}{\underline{\lambda}\left(C Q C^{T}\right)}<1,
\end{aligned}
$$

where $\bar{\sigma}(\cdot)$ and $\underline{\sigma}(\cdot)$ denote for largest and smallest singular values, respectively. Then, (14) is obtained from (16). This completes the proof.

\section{NUMERICAL EXAMPLES}

In this section, the utility of the propsed approaches for static output stabilization is demonstrated through two numerical examples.

Example 1: Consider the system presented in [5] with state-space realization

$$
A=\left(\begin{array}{llll}
0 & 0 & 0 & 0 \\
0 & 0 & 0 & 0 \\
2 & 0 & 1 & 2 \\
0 & 2 & 2 & 1
\end{array}\right), B=\left(\begin{array}{ll}
1 & 0 \\
0 & 1 \\
0 & 0 \\
0 & 0
\end{array}\right), C^{T}=\left(\begin{array}{cc}
4 & -2 \\
-3 & 2 \\
3 & -1 \\
0 & 2
\end{array}\right)
$$

The eigenvalues of $\mathrm{A}$ are located at $\{-1,0,0,3\}$. By using the Algorithm 1 with $\varepsilon_{i, j}=10^{-6}$, for $i=1,2$ and $j=1,2$, we obtain $\alpha^{*}=-0.2394$ and the resulting static output feedback gain

$$
F=\left(\begin{array}{ll}
-27.361 & -35.398 \\
-49.781 & -67.773
\end{array}\right)
$$

The closed-loop eigenvalues are $\{-2.077 \pm j 1.395$, $-9.347 \pm j 18.020\}$. On the other hand, by the LMI conditions presented in Proposition 2 for $\varepsilon=10^{-6}$, we have $\xi^{*}=1.202 \times 10^{5}$ and the static output feedback gain is

$$
F=\left(\begin{array}{cc}
-7.907 & -11.137 \\
-10.889 & 15.968
\end{array}\right)
$$


The obtained closed-loop system is stable as well with eigenvalues located at $\{-2.425 \pm j 7.889,-0.888 \pm$ j1.125\}.

Example 2: Consider the satellite example presented in [1] with dynamic equation

$$
\left\{\begin{array}{l}
J_{1} \ddot{\theta}_{1}+f\left(\dot{\theta}_{1}-\dot{\theta}_{2}\right)+k\left(\theta_{1}-\theta_{2}\right)=T \\
J_{2} \ddot{\theta}_{2}+f\left(\dot{\theta}_{2}-\dot{\theta}_{1}\right)+k\left(\theta_{2}-\theta_{1}\right)=0
\end{array}\right.
$$

where $\theta_{1}$ and $\theta_{2}$ are the attitude angles for the main body and the instrumentation module, and $T$ is the control torque. The state-space system matrices for $x=\left(\theta_{1}, \theta_{2}, \dot{\theta}_{1}, \dot{\theta}_{2}\right)^{T}, y=\left(\theta_{1}, \theta_{2}\right)^{\mathrm{T}}, u=T$, are

$$
\begin{aligned}
A & =\left(\begin{array}{cccc}
0 & 0 & 1 & 0 \\
0 & 0 & 0 & 1 \\
-k / J_{1} & k / J_{1} & -f / J_{1} & f / J_{1} \\
k / J_{2} & -k / J_{2} & f / J_{2} & -f / J_{2}
\end{array}\right), \\
B & =\left(\begin{array}{c}
0 \\
0 \\
1 / J_{1} \\
0
\end{array}\right), C^{T}=\left(\begin{array}{ll}
1 & 0 \\
0 & 1 \\
0 & 0 \\
0 & 0
\end{array}\right) .
\end{aligned}
$$

For the parameter values $J_{1}=1, J_{2}=0.1, k=$ $0.2450, f=0.0188$, the Algorithm 1 with $\varepsilon=10^{-6}$ gives $\alpha^{*}=-3.502 \times 10^{-4}$ and static output feedback gain $F=$ [-9.680 -5.513]. The obtained closed-loop eigenvalues are located at $\{-0.003 \pm j 3.129,-0.065 \pm j 0.657\}$. If the conditions of Proposition 2 are utilized for given $\varepsilon$ $=10^{-6}$, we have $\xi^{*}=0.480$ and the stabilizing static output feedback gain $F=\left[\begin{array}{ll}0.035 & -0.035\end{array}\right]$. The resulting closed-loop eigenvalues are $\{-0.0004,-0.0789,-0.063$ $\pm j 0.666\}$.

\section{CONCLUSION}

In this paper, we have presented a convex condition for the problem of static output feedback stabilization. This condition is expressed in terms of a matrix inequality subject to an equality constraint. To facilitate the LMI construction for the proposed convex condition, an iteratively numerical construction algorithm is introduced and a sufficient condition is presented as well. The numerical examples demonstrated the utility of the proposed approaches for static output feedback stabilization.

\section{REFERENCES}

1. Biernacki, R.M., Hwang, H., and Battacharyya, S.P., "Robust Stability with Structured Real Parameter
Perturbations," IEEE Trans. Auto. Control, Vol. 32, pp. 495-506 (1987).

2. Blondel, V., Gevers, M., and Lindquist, A., "Survey on the State of Systems and Control," Euro. J. Control, Vol. 1, pp. 5-23 (1995).

3. Boyd, S., El Ghaoui, L., Feron, E., and Balakrishnan, V., "Linear Matrix Inequalities in System and Control Theory," SIAM, Philadelphia (1994).

4. Cao, Y.-Y., Lam, J., and Sun, Y.X., "Static Output Feedback Stabilization: An ILMI Approach," Automatica, Vol. 34, pp. 1641-1645 (1998).

5. Crusius, C.A.R. and Trofino, A., "Sufficient LMI Conditions for Output Feedback Control Problems," IEEE Trans. Auto. Control, Vol. 44, pp. 1053-1057 (1999).

6. Gahinet, P. and Ignat, A., "Low-Order $H_{\infty}$ Synthesis Via LMI," Proceedings of the 1994 American Control Conference, pp. 1499-1450 (1994).

7. Gahinet, P., Nemirovski, A., Laub, A., and Chilali, M., "The LMI Control Toolbox," The MathWorks, Inc., Natick, MA (1995).

8. Geromel, J.C., Peres, P.L.D., and Souza, S.R., "Convex Analysis of Output Feedback Control Problems: Robust Stabilization and Performance," IEEE Trans. Auto. Control, Vol. 40, pp. 997-1002 (1996).

9. Ghaoui, L. El, Oustry, F., and AitRami, M., "A Cone Complementarity Linearization Algorithm for Static Output-Feedback and Related Problems," IEEE Trans. Auto. Control, Vol. 42, pp. 1171-1176 (1997).

10. Ho, D.W.C. and Lu, G., "Robust Stabilization for a Class of Discrete-Time Nonlinear System via Output Fedback: the Unified LMI Approach," Int. J. Control, Vol. 76, pp. 105-115 (2003).

11. Iwasaki, T. and Skelton, R.E., "All Controller for the General $H_{\infty}$ Control Problem: LMI Existence Conditions and State Space Formulas," Automatica, Vol. 30, pp. 1307-1317 (1994).

12. Kucera, V. and de Souza, C., "A Necessary and Sufficient Condition for Output Feedback Stabilization," Automatica, Vol. 31, pp. 1357-1359 (1995).

13. Prempain, E. and Postlethwaite, I., "Static Output Feedback Stabilisation with $H_{\infty}$ Performance for a Class of Plants," Syst. Control Lett., Vol. 43, pp. 158-166 (2001).

14. Syrmos, V.L., Abdallah, C.T., Dorato, P., and Grigoriadis, K., "Static Output Feedback-A Survey," Automatica, Vol. 33, pp. 125-137 (1997).

15. Trofino-Neto, A. and Kucera, V., "Stabilization via Static Output Feedback," IEEE Trans. Automatic Control, Vol. 38, pp. 764-765 (1993). 